\title{
Analysis of Appropriate Methods for Assessment of Safety in Aviation
}

\author{
Jakub Kraus \\ ATM Systems Laboratory, \\ Department of Air Transport, Faculty of Transportation \\ Sciences, Czech Technical University \\ Horská 3, Praha 2, 128 03, Czech Republic \\ e-mail: kraus@fd.cvut.cz
}

\author{
Jana Ondrášková \\ Department of Air Transport \\ Department of Air Transport, Faculty of Transportation \\ Sciences, Czech Technical University \\ Horská 3, Praha 2, 128 03, Czech Republic
}

\begin{abstract}
This article discusses the methods used for the assessment of the safety of aviation. The aim is to introduce the methods, compare them and choose the one that would be most appropriate to use for airlines around the world, and which would therefore bring the biggest increase in the overall safety in aviation.
\end{abstract}

\section{Keywords-safety, aviation safety, assessment of safety, method}

\section{INTRODUCTION}

Assessment of safety in aviation is crucial area that is currently being addressed. Significant differences between the assessment of aviation safety and other modes of transport do not allow simple assessment which is formed by a single number.

For this reason special methods are implemented which can, when operated correctly, evaluate and increase the safety and thereby reduce costs for airlines.

\section{METHODS FOR SAFETY ASSESSMENT}

\section{A. INDICATE (Identifying Needed Defences In the Civil Aviation Transport Environment)}

INDICATE is a newly invented method for safety assessment for the aerospace industry. It proactively identifies weaknesses that have the greatest potential to endanger the safety of passengers. It is designed to regularly assess the company's safety defences and minimize the likelihood of accidents.

INDICATE concept shows that in the airlines there are a number of operational areas (ground staff, maintenance and flight operations), where each could bring a number of safety risks. It is therefore necessary that the company have built up defences against them. Well-designed defences provide protection against individual and organizational error. However, the integrity of the defence must be periodically checked, because it can lose their effectiveness over time. [1]

\section{B. MCDM (Multiple-criteria decision model)}

MCDM is used to analyse the relationship between dependence criteria using the decision-making process and to determine the relative weights of criteria, showing the interdependence and feedback. In formulating the criteria, five principles are taken into account: completeness, operation, decomposition, redundancy and minimum size. [3] Aviation Safety MCDM index was developed using thirteen safety criteria, divided into four dimensions. These should be independent and should be used in the analytic hierarchy process AHP so as to create the above mentioned index. In reality, however, they are rarely independent. To overcome this problem, the analytic network process ANP is used. This is the general form of AHP, which takes off the hierarchical structural constraints.

The decision-making process and valuation method (DEMATEL) is used to detect complex relationships and building a relationship structure between the criteria for measuring the safety of the airline. Hybrid model is being DEMATEL in conjunction with ANP, which is used to create a new measurement of aviation safety.

\section{AQD (Aviation Quality Database)}

AQD is a method that helps with safety integration and risk management, and it is the world's first method which combines safety audits with reporting of events in flight, maintenance and ground operations. It is a set of tools that integrates activities associated with any hazard throughout the entire organization; from management of safety, quality and protection to work safety, environmental protection and others. It provides the necessary functionality for reporting incidents / accidents, accidents assessment, analysis, investigation and monitoring of corrective measures. AQD also connects different action and causes to ensure that each completed task will solve a real problem, thus reducing the risk of recurrence.

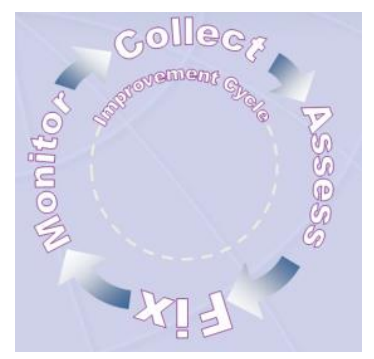

Figure 1. AQD Improvement Cycle [5] 
AQD offers total customization in key areas. For example, a company may design their own input screen for reporting, convert their existing database to AQD, so it will not lose important historical data, to continue in work with these data.

\section{ARMS (Aviation Risk Management Solutions)}

ARMS methodology is associated with three elements of ICAO SMS, its risk assessment, monitoring and measuring safety performance and change management. Therefore it develops principles that are more detailed than the more general method in ICAO SMS and SMM.

Risk identification is about the collection and analysis of operational safety data, as well as recognition of the safety issues. Such data include reports from flight events and results of safety investigations and audits. They coming into the risk assessment process as events.

Among the events may be those that require the fastest possible solution. Therefore, it is necessary to carry out an initial risk assessment immediately after their admission. This step is called events risk classification (ERC). [6]
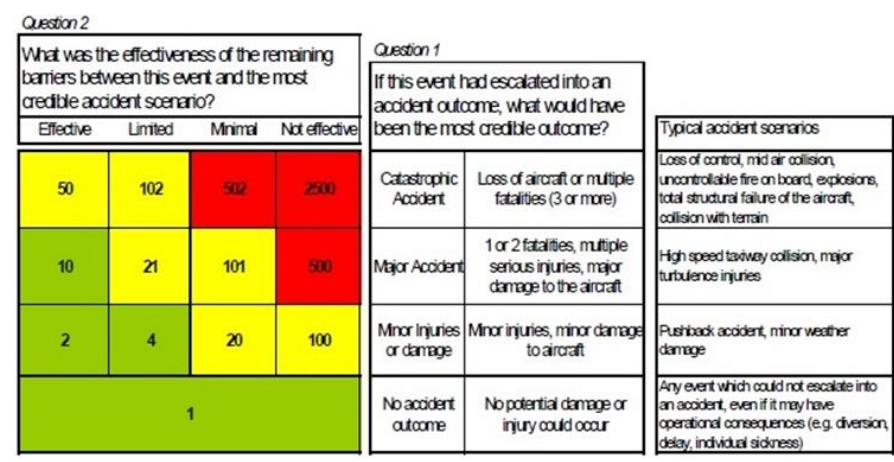

Figure 2. ARMS Risk Matrix [6]

Later in the process are identified safety problems. These will then be assessed in terms of risk. This step is called safety issue risk assessment (SIRA). [6]

Compared with the older methods of safety evaluation, ARMS brought a new perspective on safety issues in aviation. For example, the distribution of the events and issues, as well as a new risk matrix, which was unclear in the previous methods, a new structure for risk assessment of safety issues. The big advantage is that the results of the ERC and SIRA can be used individually or in combination to monitor the overall operational risk.

\section{E. IRMA (Incident Reporting Management and Analysis System)}

IRMA allows incidents to be connected with real-time information through web services. It is flexible enough to capture data from various incidents, so that each section of organization is able to manage their own types of emergencies (such as reports regarding the safety and quality of flight as well as the handling). It also contains observations of the causes and consequences of the incident, expert assessment, proofs records and delivery of messages to employees.

The great advantage of IRMA is mainly a proactive approach to risk assessment. However, most appreciated module is safety risks module and its utility for the creation of the risk matrix. It can find all the data from the incident, including the estimated and real one, and then compares proactive risk analysis and real danger.

\section{F. EMOSBOA (Quantitative evaluation method of safety based on argument)}

This method focuses on the goals that the system should achieve. By creating a link between the objectives and the evidence provided by clear evaluation method it can demonstrate that the system meets all requirements. Not only that it can evaluate safety throughout the entire process from development to usage, but it also counts many factors that have impact on safety.

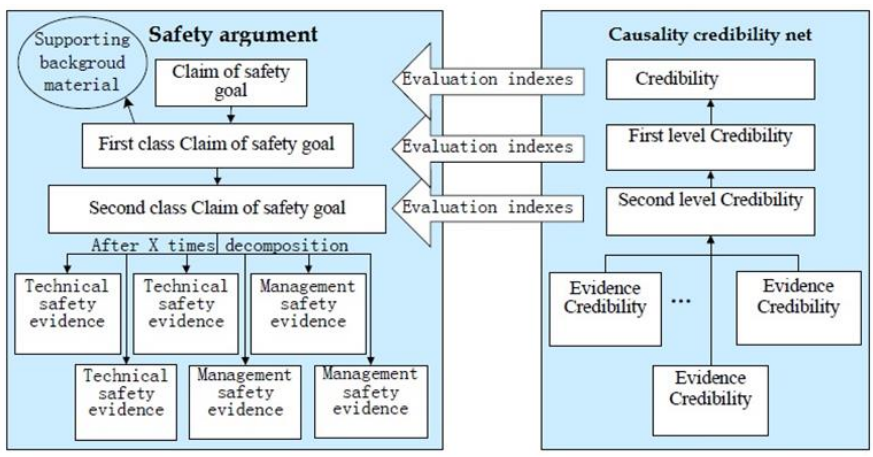

Figure 3. EMOSBOA principle [9]

EMOSBOA method proposes a new method of assessing the safety of the system, which is based on the model of argument (Goal Structuring Notation - GSN). It focuses on risk, which together with the argument provides a framework for safety assessment, which is then quantitatively judged using Bayesian networks.

\section{G. RAT (Risk Analysis Tool)}

The hazard analysis focuses on everything that could go wrong. RAT procedure firstly determines the risks that lead to specific situations, and secondly the risks are placed in sequence. This creates a dependency modelling that makes it easy to know where something is needed to improve. The method helps to document the evaluation of risks and mitigations. Its implementation and maintenance are very simple, but it is important that the RAT is used only by experienced and trained personnel.

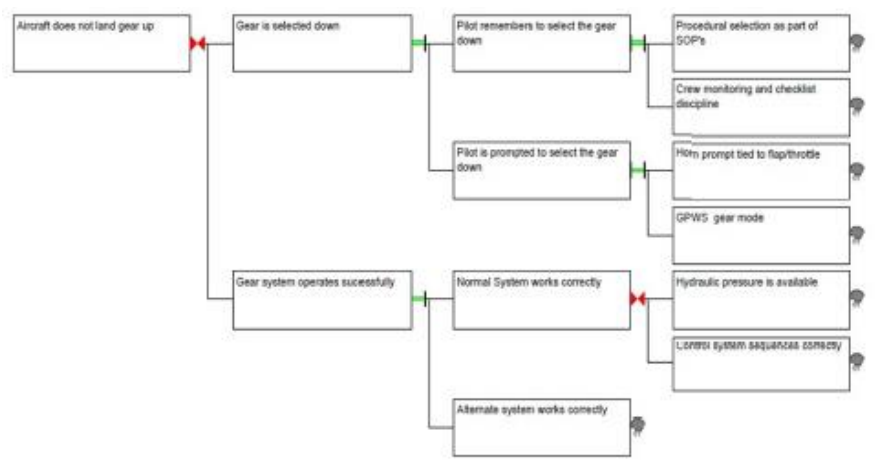

Figure 4. Example of RAT use [10] 


\section{H. TapRooT}

TapRooT's first step is to identify undesired events. This is usually an accident or incident, but it may be the process that is not functioning properly. Selection of undesired events may vary depending on investigator point of view.

After determining undesired events, the facts are arranged in chronological order. For this is used graphical representation known as SnapCharT. It consists of events (we can see what is happening), and conditions (explaining the action). After completion, SnapCharT provides valuable visual display of events, and helps to ensure that all possible information was collected.

The process of this method is systematic and provides a very well defined path for the root cause analysis. In addition, we receive data, which is perfect for monitoring the development and overall data analysis. Where this system is really good, is the analysis of complex events that represents a serious threat to air traffic. Properly conducted investigations and analysis can save time and money by combining different investigations into a process that involves all employees, which in addition provides access to relevant information arranged in a standard format.

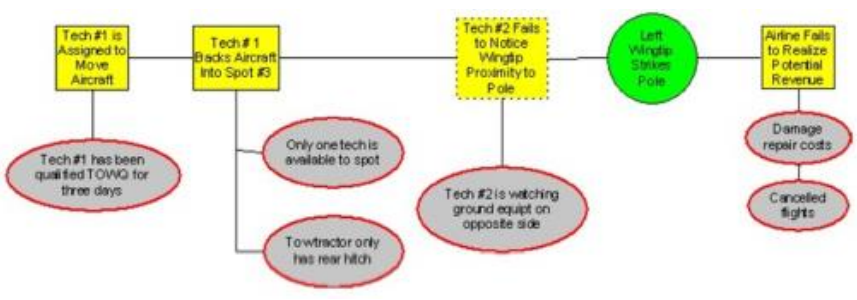

Figure 5. The Use of SnapCharT [12]

\section{COMPARISON OF METHODS FOR SAFETY ASSESSMENT}

The aim of this chapter is to compare and determine which of the above mentioned methods would be best suited for airlines and why.

In comparison, we use multi-criteria analysis. There are described criteria by which we divide each method. Assigning weights to all criteria will results in order from the most to the least appropriate method.

In best scenario, the importance of each criterion should choose every company itself, because each airlines management lead its company differently.

\section{A. The proposed criteria}

- Customized software - For clarity and monitoring of safety data has every above mentioned method its own software.

- $\quad$ Safety evaluation CONTINUOUSLY - This criterion expresses the ability of the used method to monitor the change in the overall safety of the company in time (Table Yes). If the table specifies No, it means that a safety level is assessed to a certain point in time and for further assessment will be needed to carry out a new one.
- Corrective actions monitoring - In order to monitor the improvement of the safety situation, some of the methods have (Yes) function of monitoring corrective measures.

- $\quad$ Data collection from employees (REPORTS) - Report is a message from employee who usually writes a complaint to various complications in the work environment. Yes in the table means that the method takes into account the reports and work with them.

- Distribution of final reports to employees - After being some of the procedures evaluated as unsuitable in the company, there are introduced new ones. With such changes should be employees familiar, so it is appropriate that the software is able to send new information via e-mail.

- Hazard Assessment (IN TABLES) - Some companies use methods that do not evaluate the safety, but the level of hazard. If it is specified Yes in the method, it uses a table divided to the Consequences \ Probability, and by the classification of the situation progresses further.

- Identifying Barriers - As barriers can be identified different actions which prevent incidents and accidents. It may be new regulation or change of it, or the introduction of new procedures.

- Hazard Assessment (TOTAL) - In the overall hazard assessment, the method tries to find the weakest part of the system, which further solves. It is therefore the opposite of safety evaluation.

- Data collection from employees (QUESTIONNARIES) - To collect the data necessary for the performing of the method can be used questionnaires that can be pointed to specific area as required by the company.

- Application in airlines - If the method has specified Yes, it means that it has been already tested in aviation company and proven so that it is used in normal operation.

- Safety evaluation DIRECTLY - Safety evaluation directly means that after applying a method we obtain a specific value, such a safety in percentage. In such cases there are the use of mathematical calculations. If this is done indirectly, we receive no exact value, but for example report about the overall safety.

- Use of mathematical calculations - In some cases it is necessary to obtain the evaluation from mathematical calculations. It uses the probabilities of the various variables or matrices. 
TABLE I. METHODS COMPARISON (SOURCE: AUTHORS)

\begin{tabular}{|c|c|c|c|c|c|c|c|c|}
\hline Criteria $\backslash$ Methods & INDICATE & MCDM & AQD & ARMS & IRMA & EMOSBOA & RAT & TapRoot \\
\hline Customized software & Yes & Yes & Yes & Yes & Yes & Yes & Yes & Yes \\
\hline Safety evaluation CONTINUOUSLY & Yes & No & Yes & No & Yes & No & No & No \\
\hline Corrective actions monitoring & Yes & Yes & Yes & Yes & Yes & No & No & No \\
\hline Data collection from employees (REPORTS) & Yes & Yes & Yes & Yes & Yes & No & No & No \\
\hline Distribution of final reports to employees & Yes & No & Yes & No & Yes & No & No & No \\
\hline Hazard assessment (IN TABLES) & No & No & Yes & Yes & $?$ & No & No & No \\
\hline Identifying Barriers & Yes & No & No & Yes & No & No & Yes & Yes \\
\hline Hazard assessment (TOTAL) & No & No & No & Yes & $?$ & No & Yes & Yes \\
\hline $\begin{array}{l}\text { Data collection from employees } \\
\text { (QUESTIONNAIRES) }\end{array}$ & No & Yes & No & No & No & No & No & No \\
\hline Application in airlines & Yes & Yes & Yes & Yes & Yes & No & Yes & Yes \\
\hline Safety evaluation DIRECTLY & No & Yes & No & No & No & Yes & No & No \\
\hline Use of mathematical calculations & No & Yes & No & No & No & Yes & Yes & No \\
\hline
\end{tabular}

\section{B. Assessment of table Methods comparison and assigning weights for criteria}

Each above mentioned method is different, based on a different principle. Therefore it cannot be said with certainty what is better for determining the safety and what worse, so that a greater number of Yes for method does not automatically mean better option for the company.

We have not found an answer to two criteria for IRMA method and therefore they are marked with question marks.
To be able to find the most suitable method (in our judgment), a weight is assign to criteria. These are assigned in descending order from top to bottom, so that the first two criteria have the same 11 points and the final one have one point.

According to assigned Yes / No in Table I, we rate each method and from this rating emerge the best one.

TABLE II. Evaluation OF THE Methods (SOURCE: Authors)

\begin{tabular}{|c|c|c|c|c|c|c|c|c|}
\hline Criteria $\backslash$ Methods & INDICATE & MCDM & AQD & ARMS & IRMA & EMOSBOA & RAT & TapRoot \\
\hline Customized software & 11 & 11 & 11 & 11 & 11 & 11 & 11 & 11 \\
\hline Safety evaluation CONTINUOUSLY & 11 & 0 & 11 & 0 & 11 & 0 & 0 & 0 \\
\hline Corrective actions monitoring & 10 & 10 & 10 & 10 & 10 & 0 & 0 & 0 \\
\hline Data collection from employees (REPORTS) & 9 & 9 & 9 & 9 & 9 & 0 & 0 & 0 \\
\hline Distribution of final reports to employees & 8 & 0 & 8 & 0 & 8 & 0 & 0 & 0 \\
\hline Hazard assessment (IN TABLES) & 0 & 0 & 7 & 7 & $?$ & 0 & 0 & 0 \\
\hline Identifying Barriers & 6 & 0 & 0 & 6 & 0 & 0 & 6 & 6 \\
\hline Hazard assessment (TOTAL) & 0 & 0 & 0 & 5 & $?$ & 0 & 5 & 5 \\
\hline $\begin{array}{c}\text { Data collection from employees } \\
\text { (QUESTIONNAIRES) }\end{array}$ & 0 & 4 & 0 & 0 & 0 & 0 & 0 & 0 \\
\hline Application in airlines & 3 & 3 & 3 & 3 & 3 & 0 & 3 & 3 \\
\hline Safety evaluation DIRECTLY & 0 & 2 & 0 & 0 & 0 & 2 & 0 & 0 \\
\hline Use of mathematical calculations & 0 & 1 & 0 & 0 & 0 & 1 & 1 & 0 \\
\hline TOTAL & $\mathbf{5 8}$ & $\mathbf{4 0}$ & $\mathbf{5 9}$ & $\mathbf{5 1}$ & $\mathbf{5 2}$ & $\mathbf{1 4}$ & $\mathbf{2 6}$ & $\mathbf{2 5}$ \\
\hline
\end{tabular}

\section{Conclusion for Comparison of Methods}

From Table II. it is clear that AQD reached the best evaluation. In second place is INDICATE and the third one is
IRMA. IRMA could be even better, if we could get more information about it. On the contrary, as the most inconvenient method proved to be EMOSBOA. 
Success of method AQD is mainly in a simple and clear software to which contribute employees themselves, then its continuously evaluation of safety and also the distribution of all safety changes to anyone who needs them for their work. At the same time this method is the most widely used in the airlines worldwide.

\section{CONCLUSION}

Aviation safety is an area where can still be found the room for improvement, but each improvement is more difficult than the previous one. The use of systematic methods for evaluating and improving safety is one way to achieve better level of safety in aviation.

The shown comparison of methods can be used when deciding which method to implement.

\section{ACKNOWLEDGMENT}

This paper was supported by the Grant Agency of the Czech Technical University in Prague, grant No. SGS14/212/OHK2/3T/16.

\section{REFERENCES}

[1] INDICATE. [online]
<skybrary.aero/bookshelf/books/1133.pdf>

Available at:

[2] EDKINS, G. D., John S. STRONG a C. Kurt ZORN. The INDICATE safety program: evaluation of a method to proactively improve airline safety performance. Safety Science [online]. 1998, vol. 30, issue 3, s. 275-295 [cit. 2014-08-15]. DOI: 10.1016/S0925-7535(98)00049-6. Available at: <http://80.www.sciencedirect.com. dialog.cvut.cz/science/article/pii/S0925753598000496>

[3] LIOU, James J. H., Gwo-Hshiung TZENG a Han-Chun CHANG. Airline safety measurement using a hybrid model: evaluation of a method to proactively improve airline safety performance. Journal of Air Transport Management [online]. 2007, vol. 13, issue 4, s. 243-249 [cit. 2014-08-15]. DOI: 10.1016/j.jairtraman.2007.04.008 Available at: <http://ac.els-cdn.com/S0969699707000415/1-s2.0-

S0969699707000415-main.pdf?_tid=695c1c1a-3665-11e4-9661

00000aab0f6b\&acdnat $=1410077171 \_1 d 1$ eb4bb1c8f3c705806fd1292a57 1d6>

[4] SUPERSTRUCTURE GROUP. Superstructure group AQD. [online] Available at: 〈http://www.superstructuregroup.com/aqd.aspx>

[5] ROGAN E. A Practical and Integrated Risk Management Solution, [online] (C) Superstructure Group Ltd, 2009. Available at: $<$ http://www.superstructuregroup.com/ Resources/AQD_Presentation.pps>

[6] ARMS WORKING GROUP. The ARMS Methodology for Operational Risk Assessment in Aviation Organisations.[online] ARMS Working Group, 2007-2010. Available at: <http://www.skybrary.aero/bookshelf/books/1141.pdf>

[7] ARMS GROUP, ARMS Methodology for Risk Assessment, [online] $2010 . \quad$ Available at: <http://www.skybrary.aero/index.php/ARMS_Methodology_for_Risk_ Assessment>

[8] INTELEX.COM, intelex.com. [online] Available at: <http://www.intelex.com/Virgin_Atlantic_Airways_Ltd_C1637client.aspx>

[9] ZHANG D., LU M., WU N. A quantitative evaluation method of safety based on argument. [online] China, 2014. Available at: <http://jocpr.com/vol6-iss3-2014/JCPR-2014-6-3-645-651.pdf>

[10] SAVAGE J. The Risk Analysis Tool at British Airways. [online] British Airways Safety Services, 1998. Available at: <http://flightsafety.org/files/analysis_tools.pdf>
[11] TAPROOT, About TapRooT® [online] Knoxville, Tennessee, C Copyright 2009 - 2014. Available at: <http://www.taproot.com/products-services/about-taproot>

[12] CAWBY T. TapRooT $\odot$ Root Cause Analysis Tool at Alaska Airlines. [online] Alaska Airlines, 2004. Available at: <http://flightsafety.org/files/analysis_tools.pdf> 